\begin{tabular}{|c|c|}
\hline Title & A 3-D Topology Optimization of Magnetic Cores for Wireless Power Transfer Device \\
\hline Author(s) & Otomo, Y oshitsugu; Igarashi, Hajime \\
\hline Citation & $\begin{array}{l}\text { IEEE Transactions on Magnetics, 55(6), } 8103005 \\
\text { https://doi.org/10.1109// MA G.2019.2900744 }\end{array}$ \\
\hline Issue Date & $2019-06$ \\
\hline Doc URL & http://hdl.handle.net/2115/74694 \\
\hline Rights & $\begin{array}{l}\text { (c) } 2019 \text { IEEE. Personal use of this material is permitted. Permission from IEEE must be obtained for all other uses, in } \\
\text { any current or future media, including reprinting/republishing this material for advertising or promotional purposes, } \\
\text { creating new collective works, for resale or redistribution to servers or lists, or reuse of any copyrighted component of } \\
\text { this work in other works. }\end{array}$ \\
\hline Tyре & article (author version) \\
\hline File Information & Full_paper_WPT_final_submission.pdf \\
\hline
\end{tabular}

Instructions for use 


\title{
A 3-D Topology Optimization of Magnetic Cores for Wireless Power Transfer Device
}

\author{
Yoshitsugu Otomo ${ }^{1}$, and Hajime Igarashi ${ }^{1}$, Member, IEEE \\ ${ }^{1}$ Graduate School of Information Science and Technology, Hokkaido University, Sapporo, 060-0814, Japan
}

\begin{abstract}
This paper presents the topology optimization of the magnetic core for a wireless power transfer (WPT) device. In this optimization, the coil and magnetic-core shapes for WPT are represented by the Gaussian basis functions. They are sequentially optimized using the genetic algorithm and 3D finite element analysis so that the coupling coefficient of the WPT device is maximized. Moreover, a robust optimization method is proposed to keep the interlinkage flux as large as possible against misalignment in the coils. It is shown by computational and experimental results that the optimized device outperforms the conventional devices.
\end{abstract}

Index Terms - Genetic algorithm, Misalignment, Robust optimization, Topology optimization, Wireless power transfer.

\section{INTRODUCTION}

$\mathrm{T}$ HE use of wireless power transfer (WPT) for electric vehicles (EVs) and various home appliances is expected to expand rapidly [1]-[7]. In order to improve the efficiency of a WPT device, magnetic cores are introduced in the vicinity of the transmitting and receiving coils. The amount of the magnetic core has to be made as small as possible to reduce the cost and size without deteriorating the efficiency. The magnetic core should be, therefore, carefully designed considering these factors. For such magnetic cores, bar shaped and H-shaped magnetic cores have been proposed [1, 2]. In addition, design of the transmitting and receiving coils is also very important for the efficiency of a WPT system. For this reason, several coils with, for example, double-D (DD), double-DQ and circular shapes, have been proposed [3]-[5]. In the design of WPT devices, optimal shapes have been pursued in the assumed parameter space. It is, however, not always possible to set suitable design parameters to obtain satisfactory results.

In contrast to the parameter optimization [6]-[8], the topology optimization does not require introduction of design parameters. In particular, the on/off method based on the normalized Gaussian network (NGnet) with aid of the genetic algorithm (GA) and 2D finite element method (FEM) has been shown effective for the optimization of the magnetic core of a rotating machine [9]. In this work, we propose a two-step topology optimization method for WTP devices in which the coil and magnetic-core shapes are optimized sequentially using GA and 3D FEM. Moreover, we propose a novel optimization method to make the optimal solutions robust against misalignment in the coils. The WPT device with the optimized magnetic core is manufactured to make experimental validation of the optimization method.

Manuscript received November 8, 2018; revised January 23, 2019; accepted February 18, 2019. Corresponding author: Y. Otomo.

Color versions of one or more of the figures in this paper are available online at http://ieeexplore.iee.org.

Digital Object Identifier (inserted by IEEE).

\section{OPTIMIZATION METHOD}

\section{A. Topology Optimization of Magnetic Core}

In the topology optimization of the magnetic core of a WPT device using NGnet which is schematically shown in Fig. 1, the material attribute in the design region $\Omega_{\text {core }}$ is determined from the value of the shape function defined by

$$
y(\boldsymbol{x})=\sum_{i=1}^{N} w_{i} b_{i}(\boldsymbol{x})
$$

where $w_{i}$ and $N$ denote the weighting coefficient and number of Gaussian functions, respectively. Moreover, $b_{i}(\boldsymbol{x})$ is the normalized Gaussian function given by

$$
\begin{aligned}
& b_{i}(\boldsymbol{x})=G_{i}(\boldsymbol{x}) / \sum_{j=1}^{N} G_{j}(\boldsymbol{x}) \\
& G_{i}(\boldsymbol{x})=\frac{1}{(2 \pi)^{\frac{D}{2}} \sigma^{D}} \exp \left\{-\frac{1}{2 \sigma^{2}}\left|\boldsymbol{x}-\boldsymbol{x}_{i}\right|^{2}\right\}
\end{aligned}
$$

where $\sigma, D$ and $\boldsymbol{x}_{i}$ denote the standard deviation, dimension of $\Omega_{\text {core }}$ and center of Gaussian basis. Though we assume here that $D=2$ for easiness in experimental validation, there are no difficulties in extension to 3D cores. The material attribute $M_{e}$ of finite element $e$ in $\Omega_{\text {core }}$ is determined from

$$
M_{e}=\left\{\begin{array}{cc}
\text { ferrite } & y(\boldsymbol{x}) \geq 0 \\
\text { air } & y(\boldsymbol{x})<0
\end{array}\right.
$$

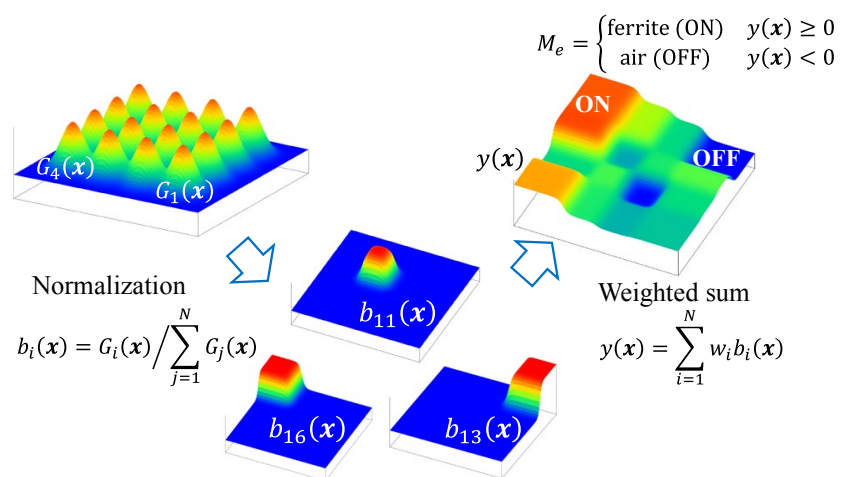

Fig. 1. On/off method using normalized Gaussians (2D example). 
In the optimization, $\boldsymbol{w}=\left\{w_{i} \mid i=1,2, \ldots, N\right\}$ is determined so as to maximize the objective function by the micro genetic algorithm $(\mu \mathrm{GA})[10]$ subjected to given constraints. Namely, the topology optimization is reduced to the parameter optimization with respect to the vector $\boldsymbol{w}=\left[w_{1}, w_{2}, \ldots, w_{N}\right]^{\mathrm{t}}$.

\section{B. Topology Optimization of Coil Shape}

In this work, the coil shape is optimized prior to the optimization of the magnetic core. To find the coil shape which maximizes the net magnetic flux interlinked with the receiving coil, we introduce a new method in which the current-density distribution $\boldsymbol{J}(\boldsymbol{x})$ is optimized. Because it is difficult to directly determine the current density satisfying current continuity $\nabla$. $\boldsymbol{J}(\boldsymbol{x})=0$, we introduce the current vector potential $\boldsymbol{T}(\boldsymbol{x})$ satisfying $\boldsymbol{J}(\boldsymbol{x})=\nabla \times \boldsymbol{T}(\boldsymbol{x})$. Then, the distribution of $\boldsymbol{T}(\boldsymbol{x})$ is determined using the NGnet-based optimization. Although it is possible to design $3 \mathrm{D}$ coil shape using the proposed method in principle, we consider here the 2D design region $\Omega_{\text {coil }}$ for a coil for simplicity. Assuming that the current density lies on the $x-y$ plane, $\boldsymbol{J}(\boldsymbol{x})=\left[J_{x}(\boldsymbol{x}), J_{y}(\boldsymbol{x}), 0\right]^{\mathrm{t}}$ is computed from $\boldsymbol{T}(\boldsymbol{x})=$ $\left[0,0, T_{z}(\boldsymbol{x})\right]^{\mathrm{t}}$. To find current density localized in a coil, we modify the shape function (1) as follows:

$$
T_{z}(\boldsymbol{x})=\alpha \tanh \left\{\sum_{i=1}^{N} w_{i} b_{i}(\boldsymbol{x})\right\} \quad\left(-\alpha \leq T_{z}(\boldsymbol{x}) \leq \alpha\right)
$$

where $\alpha$ denotes a constant. In a way similar to the optimization of the magnetic core shape, the weighting coefficient $\boldsymbol{w}$ is determined so as to maximize an objective function by $\mu \mathrm{GA}$.

\section{Naive On/Off Method}

For comparison, the magnetic core is also optimized by a naive on/off method [11], which is schematically shown in Fig. 2. In this on/off method, the material attribute of a finite element in $\Omega_{\text {core }}$ is directly optimized by $\mu$ GA. Since this method tends to fall into a checkerboard-like complicated material distribution as shown in Fig. 2, average smoothing is performed in such a way that the material attribute of element $e$ is replaced with the average of the neighboring attributes as follows:

$$
M_{e}=\text { air if } \quad \Sigma_{e^{\prime} \in N(e)} g\left(M_{e^{\prime}}=\text { ferrite }\right)<3
$$

where $N(e)$ and $g$ denote the neighbor of element $e$ and the function defined by $g(*)=1(0)$ if $*$ is true (false).

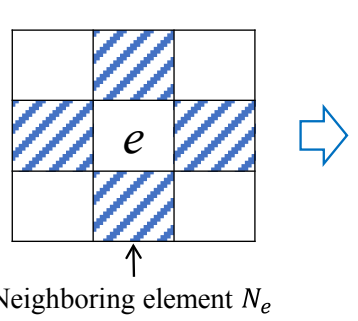

Fig. 2. Average smoothing for conventional on/off method

\section{OPTIMIZATION PROBLEM}

\section{A. Topology Optimization of Coil}

In this optimization, we consider a WPT device for EVs. In the first step, the coil shape is optimized using the method mentioned in II-B. The optimization problem is defined by

$$
\max _{w} F_{1}(\boldsymbol{w}), \quad F_{1}(\boldsymbol{w})=\Phi_{\mathrm{z}}(\boldsymbol{w})
$$

where $\Phi_{\mathrm{z}}(\boldsymbol{w})$ denotes the magnetic flux in $z$-direction across a rectangular domain $\Omega_{\Phi}$ at distance $h$ from $\Omega_{\text {coil }}$, shown in Fig. 3. Note that $\Phi_{\mathrm{z}}(\boldsymbol{w})$ is the implicit function of the weighting coefficient $\boldsymbol{w}$ in (5).

To shape the coil with mirror symmetries in $x$ and $y$ directions, 96 Gaussian functions are uniformly deployed so that the $1 / 4$ fraction of the coil design region is effectively covered the Gaussians, that is, the standard deviation is determined as $\sigma=$ $30.0 \mathrm{~mm}$ so that the Gaussians have overlaps each other.

\section{B. Topology Optimization of Magnetic Core}

In the second step posterior to the optimization of a coil, the magnetic core shape is optimized so that the core becomes as small as possible for reduction of cost and size, and simultaneously its efficiency is kept as high as possible even though misalignment between the transmitting and receiving coils exists. To obtain the optimal core shape which has a good tolerance to the misalignment, we maximize the magnetic coupling for several vertical misalignment patterns. Thus, the optimization problem is defined by

$$
\max _{w} F_{2}(\boldsymbol{w}), \quad F_{2}(\boldsymbol{w})=\frac{1}{N_{\mathrm{P}}} \sum_{i=1}^{N_{\mathrm{p}}} k_{i}(\boldsymbol{w}),
$$

sub. to $V_{\text {core }} \leq V_{0}$

where $k_{i}(\boldsymbol{w})\left(i=1,2, \ldots, N_{\mathrm{P}}\right), V_{\text {core }}$ and $V_{0}$ denote the coupling coefficient for the $i$-th misalignment pattern and total volume of the optimized core and given upper limit, respectively. In (8), the simple average of $k_{i}$ is considered, while we can modify the problem by introducing different weighting coefficient for $k_{i}(\boldsymbol{w})$. Assuming that the transmitting and receiving coils have the same self-inductance, the coupling coefficient $k_{i}(\boldsymbol{w})$ is obtained from the FE analysis as follows:

$$
k_{i}(\boldsymbol{w})=\frac{\int_{\Omega_{\text {coil } 2}} \boldsymbol{A}_{i}(\boldsymbol{w}) \cdot \boldsymbol{J}_{2} d \Omega}{\int_{\Omega_{\text {coil } 1}} \boldsymbol{A}_{i}(\boldsymbol{w}) \cdot \boldsymbol{J}_{1} d \Omega}
$$

where $\boldsymbol{A}_{i}(\boldsymbol{w}), \boldsymbol{J}_{1}$ and $\boldsymbol{J}_{2}$ denote the vector potential for $i$-th misalignment pattern and unit vectors parallel to the currents along the transmitting and receiving coils, respectively. Note that the vector potential $\boldsymbol{A}_{i}(\boldsymbol{w})$ is the implicit function of the weighting coefficient $\boldsymbol{w}$.

The design region $\Omega_{\text {core }}$ for the magnetic core adjacent to the coils is shown in Fig. 6. TABLE II summarizes the coil parameters. To shape the transmitting and receiving coils with mirror symmetries in $x, y$ and $z$ directions, 98 Gaussian functions whose standard deviation $\sigma=15.0 \mathrm{~mm}$ are uniformly deployed in the $1 / 4$ fraction in $\Omega_{\text {core }}$. 


\section{OPTIMIZATION RESULTS}

\section{A. Coil Shape}

TABLE I summarizes the model parameters. To solve optimization problem (7) using $\mu \mathrm{GA}$, the number of individuals is set to 5 and the evolution process is continued for 400 generations. It takes about 70 minutes to obtain the optimization results using the Intel Xeon $C P U(3.2 \mathrm{GHz}, 5$ cores $)$. It is remarked that $90 \%$ of the computing time is spent for the FE analysis.

The optimized current distribution is shown in Fig. 4 (a), where its maximum value is set to $1.0 \times 10^{4} \mathrm{~A} / \mathrm{m}^{2}$ by (5). We can see that the resultant current concentrates in a ring-shaped region colored by red. The ring-shaped region is approximated by a circular ring shown in Fig. 4 (b). Note that we obtain the circular coil without any prescribed parameters. If the target domain $\Omega_{\Phi}$ is changed, a different optimal current distribution would be obtained. To verify the effectiveness of this result, we compute the values of $\Phi_{\mathrm{z}}$ by changing the inner or outer radius of the ring coil obtained by the optimization. The fluxes normalized by the flux of the optimized coil, $\Phi_{\text {opt }}$, are plotted in Fig. 5. We can see that the obtained coil has the largest flux. We next design the magnetic core for the optimized coil below.

TABLE I

SPECIFICATIONS OF OPTIMIZATION MODEL FOR COIL DESIGN

\begin{tabular}{ccc}
\hline \hline & Coil design region & Target domain \\
\hline Width (x-direction) & $900 \mathrm{~mm}$ & $60 \mathrm{~mm}$ \\
Depth $(y$-direction) & $300 \mathrm{~mm}$ & $20 \mathrm{~mm}$ \\
Height (z-direction) & $3.5 \mathrm{~mm}$ & $3.5 \mathrm{~mm}$ \\
\hline \hline
\end{tabular}

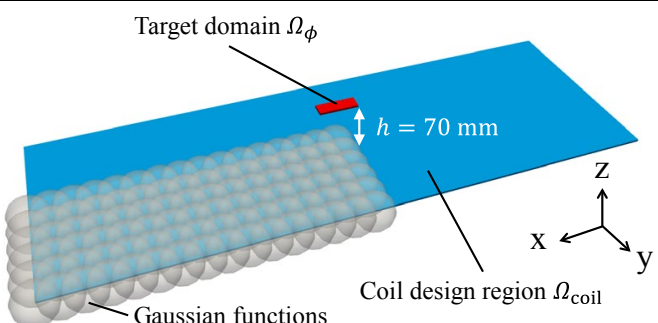

Fig. 3. Optimization model for coil design

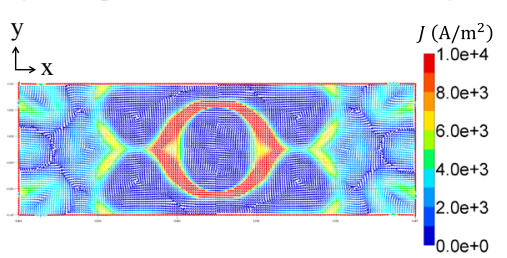

(a) Optimized current density

Fig. 4. Optimized current density and optimal coil

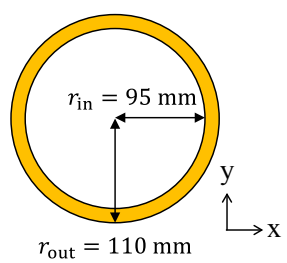

(b) Optimal coil
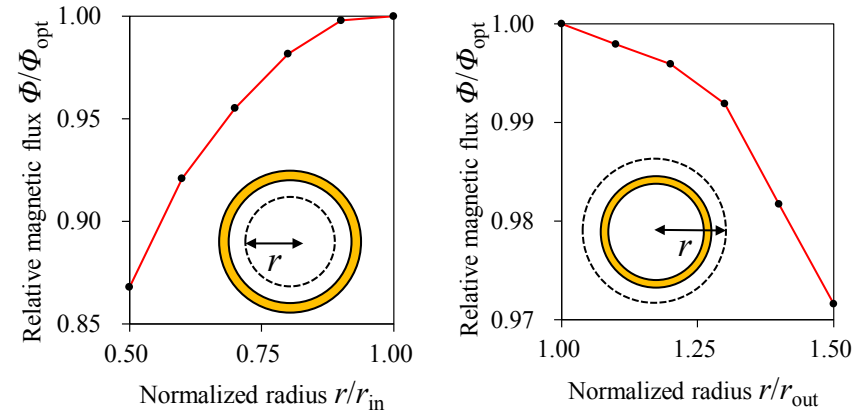

(a) Change in inner radius

(b) Change in outer radius

Fig. 5. Comparison results of relative magnetic flux in target domain

\section{B. Magnetic Core shapes}

It is assumed that misalignment in the forward direction $(y$ direction) is easily limited by a wheel stopper, whereas the misalignment in the lateral direction ( $x$-direction) cannot be limited. Under this assumption, we consider the lateral misalignments for problem (8). Assuming rather wide tolerance is required with respect to the lateral misalignment, we consider three different misalignments $0 \mathrm{~mm}, 80 \mathrm{~mm}, 160 \mathrm{~mm}$, which are indexed by $i=1,2,3$ in (8), respectively. Problem (8) is solved using $\mu \mathrm{GA}$ under the same condition as that for the

TABLE II

SPECIFICATIONS OF WPT COILS

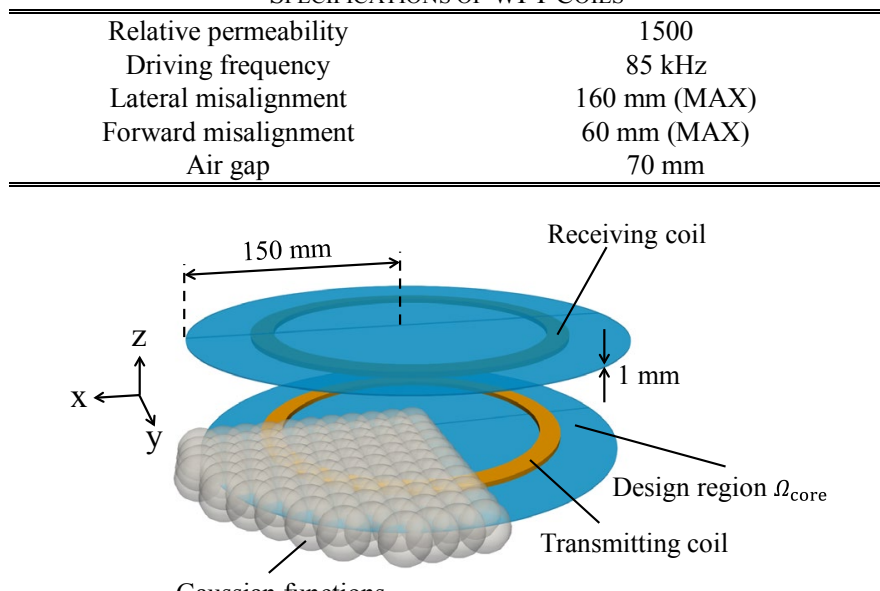

Gaussian functions

Fig. 6. Optimization model for magnetic core design

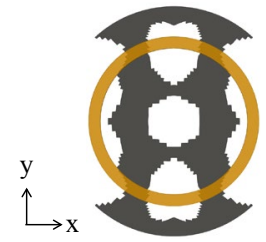

(a) Optimized (NGnet) $V_{\text {core }}=2.99 \times 10^{-5} \mathrm{~m}^{3}$ Fig. 7. WPT cores

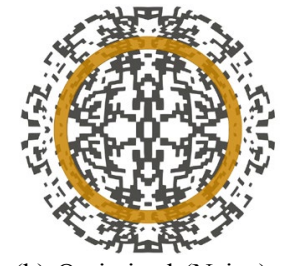

(b) Optimized (Naive) $V_{\text {core }}=2.85 \times 10^{-5} \mathrm{~m}^{3}$

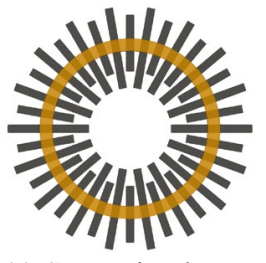

(c) Conventional $V_{0}=3.06 \times 10^{-5} \mathrm{~m}^{3}$

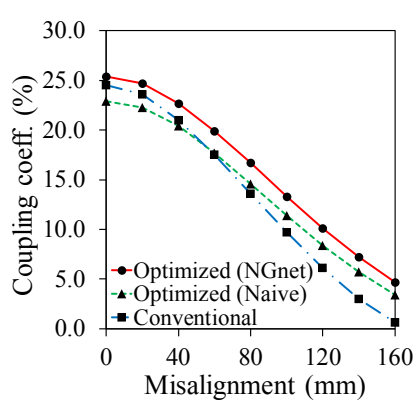

(a) Lateral $(\mathrm{x})$ misalignment

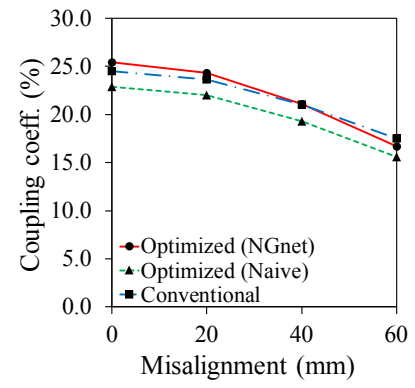

(b) Forward (y) misalignment Fig. 8. Coupling coefficients of each WPT core

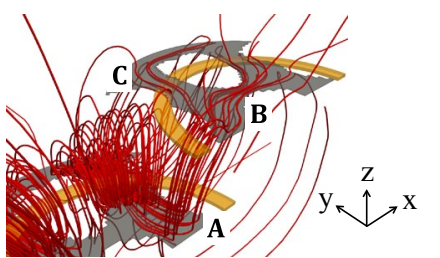

(a) Optimized (NGnet)

Fig. 9. Comparison results of flux distributions with lateral misalignment $=160 \mathrm{~mm}(1 / 2$ fraction of WPT is shown $)$

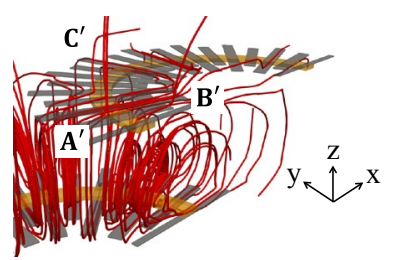

(b) Conventional 
optimization of the coil. It takes about a day to obtain the optimization results using the Intel Xeon CPU $(3.2 \mathrm{GHz}, 5$ cores).

The optimized results using NGnet and naive on/off method with the smoothing, represented by "Optimized (NGnet)" and "Optimized (Naive)", respectively, are shown in Fig. 7 (a), (b). For comparison, the bar shaped core, which is presented in [1] is also shown in Fig. 7 (c). In addition, the volume of the each WPT core is shown in Fig. 7. One of the remarkable features in the optimized result obtained by NGnet is existence of the large holes in the core. On the other hand, the naive on/off method results in the complicated magnetic core in spite of the average smoothing. Even when the smoothing is repeatedly performed, the performance cannot be improved. The coupling coefficients are plotted against the degree of misalignment in Fig. 8. We can see that the optimized WPT shown in Fig. 7 (a) keeps the relatively high coupling-coefficient value not only against lateral misalignment but also forward misalignment. The coupling coefficient of the optimized WPT in Fig. 7 (a) with misalignment of $160 \mathrm{~mm}$ is larger by about $1.3 \%$ and $4.1 \%$ than that of the optimized WPT in Fig. 7 (b) and conventional cores. To interpret the difference in the performance of the WPTs in Fig. 7 (a) and (c), the flux distributions with lateral misalignment are compared in Fig. 9. It can be seen that there are fluxes along path " $\mathrm{A}-\mathrm{B}-\mathrm{C}$ " which effectively interlink with the receiving coil in Fig. 9 (a). On the other hand, there are little linkage flux in Fig. 9 (b) because the fluxes tend to go along " $A$ ' $-B^{\prime}-C$ "' without net interlinkage.

\section{EXPERIMENTAL VALIDATION}

To verify the numerical results, the performance of optimized and conventional WPT devices is experimentally evaluated. Because it is difficult to manufacture the optimized magnetic core using bar shaped ferrite cores, we make use of the soft ferrite sheet whose relative permeability is 9-14 [12]. The manufactured WPTs are shown in Fig. 10 (a), (b). TABLE III summarizes the coil specifications. The measured coupling coefficients are plotted against the degree of misalignment in Fig. 10 (c), (d). We can see that the optimized WPT has the higher coupling coefficients than that of the conventional WPT. It is concluded form these results that the proposed method is effective to improve the WPT performance.

\section{CONCLUSION}

In this paper, we have proposed a new two-step topology optimization method based on $3 \mathrm{D}$ field computing to effectively improve the efficiency of the WPT device. It has been shown that the optimized WPT is more robust against misalignment than the other two WPTs. This result is validated by experiment. The propose method can be applied to the topology optimization of WPTs of the other types, which will be discussed elsewhere. Moreover, the multi-objective optimization of the coil and magnetic core will be studied in future.
TABLE III

SPECIFICATIONS OF EXPERIMENTAL WPT COILS
Strand radius

Number of strands

Number of turns
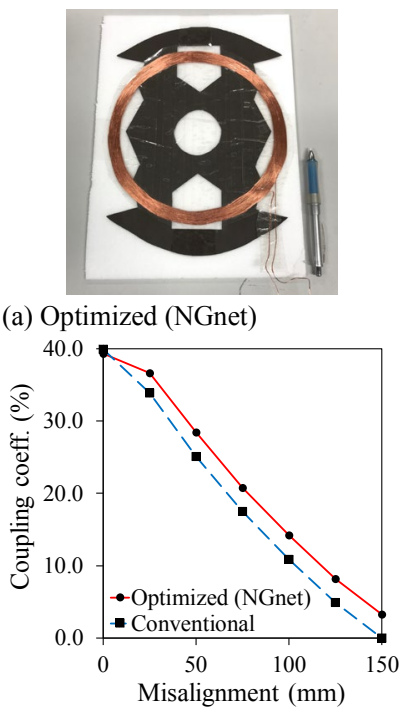

(c) Lateral (x) misalignment with air gap $=25 \mathrm{~mm}$ Fig. 10. Manufactured WPTs and measured coupling coefficients (a) Optimized (NGnet) (b) Conventional

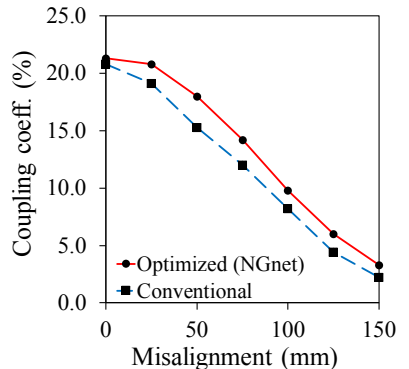

(d) Lateral (x) misalignment with air gap $=55 \mathrm{~mm}$

\section{REFERENCES}

[1] M. Budhia, G. A. Covic and J. T. Boys, "Design and Optimization of Circular Magnetic Structures for Lumped Inductive Power Transfer Systems," IEEE Trans. Power Electron., vol. 26, no. 11, pp. 3096-3108, 2011.

[2] M. Chigira, Y. Nagatsuka, Y. Kaneko, S. Abe, T. Yasuda and A. Suzuki, "Small-Size Light-Weight Transformer with New Core Structure for Contactless Electric Vehicle Power Transfer System," IEEE Energy Conversion Congress and Exposition, pp. 260-266, 2011.

[3] A. A. S. Mohamed, S. An and O. Mohammed, "Coil Design Optimization of Power Pad in IPT System for Electric Vehicle Applications," IEEE Trans. Magn., vol. 54, no. 4, Art. no. 9300405, 2018.

[4] M. Budhia, J. T. Boys, G. A. Covic and C. Y. Huang, "Development of a Single-Sided Flux Magnetic Coupler for Electric Vehicle IPT Charging Systems," IEEE Trans. Ind. Electron., vol. 60, no. 1, pp. 318-328, 2013.

[5] A. Tejeda, C. Carretero, J. T. Boys and G. A. Covic, "Ferrite-Less Circular Pad with Controlled Flux Cancelation for EV Wireless Charging," IEEE Trans. Power Electron., vol. 32, no. 11, pp. 8349-8359, 2017.

[6] T. Yilmaz, N. Hasan, R. Zane and Z. Pantic, "Multi-Objective Optimization of Circular Magnetic Couplers for Wireless Power Transfer Applications," IEEE Trans. Magn., vol. 53, no. 8, Art. no. 8700312, 2017.

[7] A. Hariri, A. Elsayed and O. A. Mohammed, "An Integrated Characterization Model and Multiobjective Optimization for the Design of an EV Charger's Circular Wireless Power Transfer Pads," IEEE Trans. Magn., vol. 53, no. 6, Art. no. 8001004, 2017.

[8] L. D. S. Coelho, V. C. Mariani, F. A. Guerra, M. V. F. da Luz and J. V. Leite, "Multiobjective Optimization of Transformer Design Using a Chaotic Evolutionary Approach," IEEE Trans. Magn., vol. 50, no. 2, Art. no. 7016504,2014

[9] T. Sato, K. Watanabe and H. Igarashi, "Multimaterial Topology Optimization of Electric Machines Based on Normalized Gaussian Network," IEEE Trans. Magn., vol. 51, no. 3, Art. no. 7202604, 2015.

[10] C. A. C. Coello and G. T. Pulido, "A Micro-Genetic Algorithm for Multiobjective Optimization,” EMO 2001, pp. 126-140, 2001.

[11] O. Sigmund and J. Petersson, "Numerical instabilities in topology optimization: A survey on procedures dealing with checkerboards, meshdependencies and local minima," Structural Optimization, vol. 16, no. 1, pp. 68-75, 1998.

[12] ONO GOMU KOUGYO, "Soft ferrite sheet," http://www.onogomu.co.jp/wp-content/themes/onogomuchild/pdf/mag02.pdf (in Japanese). 